\title{
Satellyptus: Analysis and database of microsatellites from ESTs of Eucalyptus
}

\author{
Paulo Cezar Ceresini ${ }^{1}$, Cristina Lacerda Soares Petrarolha Silva ${ }^{1,2}$, Robson Fernando Missio ${ }^{3}$, \\ Elaine Costa Souza ${ }^{1}$, Carlos Norberto Fischer ${ }^{4}$, Ivan Rizzo Guillherme ${ }^{4}$, Ivo Gregorio ${ }^{4}$, \\ Eloiza Helena Tajara da Silva ${ }^{5}$, Regina Maria Barreto Cicarelli ${ }^{6}$, Marco Túlio Alves da Silva ${ }^{6}$, \\ José Fernando Garcia ${ }^{7}$, Gustavo Arbex Avelar ${ }^{7}$, Laercio Ribeiro Porto Neto ${ }^{7}$, André Ricardo Marçon ${ }^{7}$, \\ Maurício Bacci Junior ${ }^{8}$, Danyelle Cristine Marini ${ }^{8}$ \\ ${ }^{1}$ Institute of Plant Sciences/Phytopathology, Swiss Federal Institute of Technology, ETH-Zentrum, Zurich, \\ Switzerland / Universidade Estadual Paulista 'Júlio de Mesquita Filho' (UNESP), Departamento de \\ Fitossanidade, Eng. Rural e Solos, Ilha Solteira, SP, Brazil. \\ ${ }^{2}$ Faculdades Integradas Stella Maris, Fundação Educacional de Andradina, Andradina, SP, Brazil. \\ ${ }^{3}$ UNESP, Departamento de Fitotectnia, Sócio-Economia e Tecnol. de Alimentos, Ilha Solteira, SP, Brazil. \\ ${ }^{4}$ UNESP, Departamento de Estatística, Matemática Aplicada e Computação, Rio Claro, SP, Brazil. \\ ${ }^{5}$ UNESP, Departamento de Biologia, S.J. Rio Preto, SP, Brazil. \\ ${ }^{6}$ UNESP, Departamento de Ciências Biológicas, Araraquara, SP, Brazil. \\ ${ }^{7}$ UNESP, Departamento de Apoio, Produção e Saúde Animal, Araçatuba, SP, Brazil. \\ ${ }^{8}$ UNESP, Centro de Estudos de Insetos Sociais, Rio Claro, SP, Brazil.
}

\begin{abstract}
The main goal of our research was to search for SSRs in the Eucalyptus EST FORESTs database (using a software for mining SSR-motifs). With this objective, we created a database for cataloging Eucalyptus EST-derived SSRs, and developed a bioinformatics tool, named Satellyptus, for finding and analyzing microsatellites in the Eucalyptus EST database. The search for microsatellites in the FORESTs database containing 71,115 Eucalyptus EST sequences (52.09 Mb) revealed 20,530 SSRs in 15,621 ESTs. The SSR abundance detected on the Eucalyptus ESTs database ( $29 \%$ or one microsatellite every four sequences) is considered very high for plants. Amongst the categories of SSR motifs, the dimeric (37\%) and trimeric ones (33\%) predominated. The AG/CT motif was the most frequent $(35.15 \%)$ followed by the trimeric CCG/CGG (12.81\%). From a random sample of 1,217 sequences, 343 microsatellites in 265 SSR-containing sequences were identified. Approximately $48 \%$ of these ESTs containing microsatellites were homologous to proteins with known biological function. Most of the microsatellites detected in Eucalyptus ESTs were positioned at either the 5' or 3' end. Our next priority involves the design of flanking primers for codominant SSR loci, which could lead to the development of a set of microsatellite-based markers suitable for marker-assisted Eucalyptus breeding programs.
\end{abstract}

Key words: bioinformatics tool, genetic markers, gene ontology, small sequence repeats, SSR.

Received: May 28, 2004; Accepted: June 21, 2005.

\section{Introduction}

Microsatellites, also described as polymorphic simple sequence repeats (SSRs), are widely distributed, short, tandemly repeated sequences of 1-6 nucleotides. The uniqueness and importance of SSRs arise from their multiallelic nature, codominant inheritance, relative abun-

Send correspondence to Paulo Cezar Ceresini. Institute of Plant Science/Phytopathology, Swiss Federal Institute of Technology ETH, Zentrum, Universitaetstr. 2 / LFW-B28, CH-8092, Zurich, Switzerland. E-mail: paulo.ceresini@ipw.agrl.ethz.ch. dance, extensive genome coverage and ease of detection by PCR using unique primers that flank and define the repeat (Powell et al., 1996). As a result of these characteristics, microsatellites have become the markers of choice for constructing framework genetic maps in plants.

The standard method for the development of SSR markers involves the creation of a small insert genomic library, a subsequent DNA hybridization selection and clone sequencing. Several hundreds of SSR markers in cultivated plants were developed with this methodology (Struss and 
Plieske, 1998; Ramsay et al., 2000). The growing body of genetic knowledge available in DNA and expressed sequence tag (EST) databases has provided an alternative approach for microsatellite detection. SSRs can be directly sourced in such databases, reducing the time and costs of microsatellite development. So far, identification of ESTderived microsatellites has been performed for some plant species, including grape (Scott et al., 2000), sugar cane (Cordeiro et al., 2001), wheat (Eujayl et al., 2002), rye (Hackauf and Wehling, 2002) and barley (Thiel et al., 2003).

A comparison of the repeat lengths of SSRs in clones from enriched libraries vs. SSRs derived from databases showed that the repeats found in libraries were significantly longer (Ramsay et al., 2000). However, EST-derived microsatellites offer the potential of more cost-effective data acquisition than standard libraries and other markers, such as single nucleotide polymorphisms (SNPs), restriction fragment length polymorphism (RFLP) and amplified fragment length polymorphism (AFLP). Because of their codominant inheritance, ESTs-derived SSRs are extremely useful as markers for genomic diversity and phylogenetic analyses in collections of clones presenting traits of agronomic interest as well as for genetic improvements and in building genetic linkage maps for localizing ESTs associated with genes of putative functions.

Considering the availability of increasing amounts of genetic information generated by EST sequencing in the FORESTs Project (sponsored by FAPESP - Fundação de Amparo à Pesquisa do Estado de São Paulo), the development of Eucalyptus microsatellite markers derived from EST database becomes an attractive approach. The main objective of this research was to search, characterize and catalog the Eucalyptus EST-derived SSR-motifs according to their abundance, information content, and genomic distribution. The task of finding and analyzing microsatellites in a database of sequences requires specialized knowledge and laborious human effort. This task is greatly facilitated with the utilization of bioinformatics tools for accelerating information recovery from databases. For this purpose, a database was created for cataloging Eucalyptus ESTderived SSRs, and a bioinformatics tool, named Satellyptus, was developed to help in the task of finding and analyzing microsatellites in the Eucalyptus EST database. To our knowledge, this is the first time such a broad search for microsatellites in EST-databases and subsequent characterization and cataloging of potential SSR-based markers for Eucalyptus has been attempted.

\section{Materials and Methods}

Data from EST libraries representing different species of Eucalyptus, originating from several tissues, developmental stages and environmental conditions, were analyzed for the presence of SSRs. Preliminarily, poly-A and poly- $\mathrm{T}$ sequences corresponding to poly-A tail from
Eukaryotes were removed from EST libraries, observing the inexistence of any remaining $(\mathrm{A})_{5}$ or $(\mathrm{T})_{5}$ in a $50 \mathrm{bp}$ window, both in 3' and 5' ends respectively.

In order to align and group the reads in clusters, CAP3 script (Huang and Madan, 1999) was applied to the EST libraries. The average size of sequences was around $700 \mathrm{bp}$, excluding 3' extremities of longer sequences in order to increase the quality of analysis.

SSR search was performed in a complete data bank including 71,115 EST consensus sequences from Eucalyptus (representing a total of $52.09 \mathrm{Mb}$ ) grouped by library. MISA (MIcroSAtellite identification tool) software was used in the present study (http://pgrc.ipk-gatersleben.de /misa/) (Thiel et al., 2003). This software allows the localization and identification of both perfect and composed microsatellites, the latter, in general, interrupted by mismatched bases. Sequences were considered microsatellites if they showed repeated motifs ranging from one to six nucleotides. Using this approach, to be considered a microsatellite, a sequence must present a minimum of 10 repeat units for mononucleotide motifs, 6 repeat units for dinucleotide motifs and 5 repeat units for all the motifs of larger size.

A random sample of 265 SSR-containing EST sequences was characterized according to their gene function, considering previous information on automatic BLASTX homology comparisons, available at the FORESTs database. This random sample was obtained from the complete Eucalyptus EST database by analyzing 1,217 sequences $(862 \mathrm{~kb})$ for SSR content in a partial backup dataset deposited at http://www.esalq.usp.br/ciagri (password-restricted access), which represented approximately $1.70 \%$ of the total available data. Known protein sequences producing significantly high score hits with Eucalyptus EST sequences were checked for their function against UniProt and InterPro databanks deposited at the European Bioinformatics Institute - EBI (http://srs.ebi.ac.uk/) and grouped by their gene ontology using QuickGO, a fast web-based browser of Gene Ontology data information, also available at EBI (http://www.ebi.ac.uk/ego/).

\section{The development of the Satellyptus system}

A computational system named Satellyptus was conceived and developed to aid in the task of finding and analyzing microsatellites in the Eucalyptus EST database, providing a way to integrate some bioinformatics tools and data generated along the mining and data analysis phases. The process for finding the microsatellites of interest utilizing Satellyptus is accomplished in two stages: first, a database of microsatellites is generated; the second stage consists of the analysis of this database.

The bioinformatics tool named MISA, which is a PERL5 script (Thiel et al., 2003), was utilized for identifying microsatellites in a large set of Eucalyptus EST sequences. MISA processing generates two text files: one, 
containing information relating to the microsatellites found in the sequences, and another containing statistics referring to the data analyzed.

Due to the difficulties in analyzing the great volume of data in text format generated by MISA, we developed a computational tool, called MSAT, which imports data from the MISA files to a relational database, created by our team as well. Some additional information was integrated into the microsatellite database. As the MISA script was individually run on subsets of data hierarchically grouped into distinct Eucalyptus EST libraries, extra classification fields were incorporated into the microsatellite database by which the data can be sorted by EST library and/or Eucalyptus species.

With the help of Satellyptus, it was possible to perform several types of data recovery in order to obtain more specific information. Satellyptus also includes normalization procedures that aid in the understanding of the occurrence and distribution of microsatellites in the Eucalyptus EST sequence database. For example, by using filters and classification for a given Eucalyptus EST library or species one can check for the occurrence of microsatellites in the database concerning the main motif categories detected, their nature (perfect or compound), and their size (repeat number). Other extremely useful information for selection of a particular microsatellite for genetic marker development is the relative position of microsatellites within an EST sequence (from 5' to 3' end). Therefore, by incorporating both MISA information on the location of a particular microsatellite in a given sequence and information from the Eucalyptus ESTs concerning the size (bp) of this sequence, the microsatellites were categorized and labeled as I (initial), M (middle) or E (end) to incorporate data on their relative positions within the given sequence.

Satellyptus also allows for easy and fast integration, in the relational database, of data from annotation. For example, a function table option will provide information on known protein sequences, producing significantly high score hits with Eucalyptus EST sequences containing microsatellites. This function table option contains information on the putative protein functions (which were checked against UniProt and InterPro databanks deposited at EBI) and their gene ontology (which was determined using QuickGO, also available at EBI). Figure 1 shows the steps for generation of the Eucalyptus EST-derived microsatellites database.

\section{Results}

The search for microsatellites in the complete FORESTs database containing 71,115 Eucalyptus EST sequences (representing a total of $52.09 \mathrm{Mb}$ ) revealed 20,530 SSRs in 15,621 ESTs (Table 1.A). This corresponded, on average, to one SSR-containing sequence every $3.9 \pm 1.0$ EST, or an average distance between two SSRs of approximately $2,702 \pm 365 \mathrm{bp}$. The average size of EST sequences

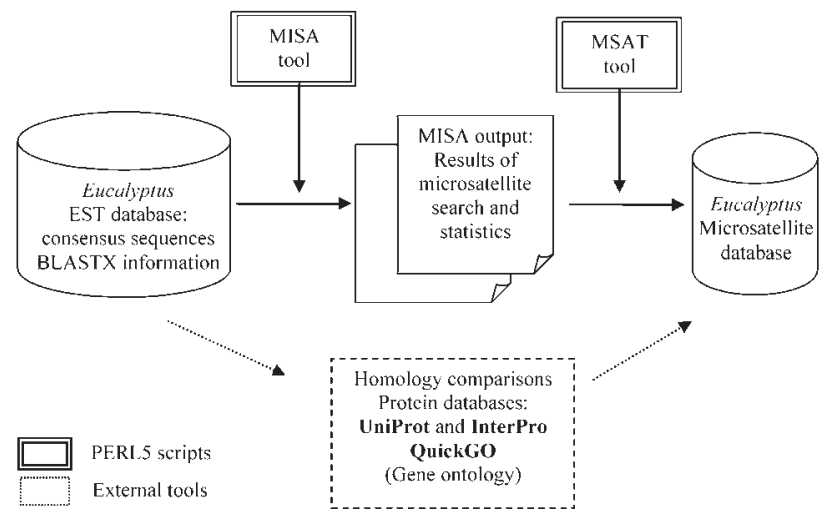

Figure 1 - The generation of the Eucalyptus EST-derived microsatellites database. $(\mathrm{EST}=$ expressed sequence tag, BLASTX = homology comparison tool, $\mathrm{MISA}=$ microsatellite identification tool, $\mathrm{MSAT}=$ computation tool which imports data from the MISA).

examined was $714 \pm 85$ bp. Approximately 3,799 EST sequences contained more than one microsatellite, while in 2,784 sequences SSRs were present in compound formation.

Similar results were observed with a random sample from the total database (Table 1.B). This random sample was obtained from the complete Eucalyptus EST database

Table 1 - MISA statistics of both complete and random sampling from the Eucalyptus EST FORESTs database.

\begin{tabular}{lc}
\hline Sampling & Observed values \\
\hline A. Complete Eucalyptus EST database & 71,115 \\
Total number of sequences examined & 52,094 \\
Total size of examined sequences (thousands of bp) & $714 \pm 85$ \\
Average size of examined EST sequences (bp) & 20,530 \\
Total number of identified SSRs & $3.9 \pm 1.00$ \\
Ratio of identified SSR: sequences examined & $2,702 \pm 365$ \\
Average distance between SSRs (bp) & 15,621 \\
Number of SSR containing sequences (A) & 3,799 \\
Number of sequences containing more than 1 SSR & 2,784 \\
Number of SSRs present in compound formation & \\
\hline B. Random sample from the total database & 1,217 \\
\hline Total number of sequences examined & 862 \\
Total size of examined sequences (thousands of bp) & $741 \pm 112$ \\
Average size of examined EST sequences (bp) & 343 \\
Total number of identified SSRs & $4.0 \pm 3.0$ \\
Ratio of identified SSR: sequences examined & $2,759 \pm 141$ \\
Average distance between SSRs (bp) & 53 \\
Number of SSR containing sequences (B) & \\
Number of sequences containing more than 1 SSR & \\
Number of SSRs present in compound formation & \\
Percentage of the total sequences containing SSR & \\
sampled [(B/A)X100] & \\
\hline
\end{tabular}


by analyzing 1,217 sequences $(862 \mathrm{~kb}$ ) for SSR content in a partial backup dataset deposited at http://mastin.ciagri.usp. br/genoma/ (USP/ESALQ/CIAGRI - Informatics Center), which represented approximately $1.70 \%$ of the total available data. A total of 265 SSR-containing sequences were identified in this sample. Fifty-nine of these sequences contained more than one SSR, whereas 43 SSRs were present in compound formation. A total of 343 microsatellites were identified. This corresponded to an average of one SSRcontaining sequence every $4.0 \pm 3.0 \mathrm{EST}$, or a distance between two SSRs of approximately $2,759 \pm 141$. The average size of EST sequences examined from this random sample was $741 \pm 112 \mathrm{bp}$.

The proportion of SSR unit sizes observed in the complete FORESTs database was not evenly distributed: 5,499 (26.79\%) were mononucleotide; 7,599 (37.01\%) dinucleotide; $6,872(33.47 \%)$ trinucleotide; 296 (1.44\%) tetranucleotide; $86(0.42 \%)$ pentanucleotide; and 178 $(0.87 \%)$ hexanucleotide (Table 2 ; Appendix Table, available upon request or at the site https://forests.esalq.usp.br; password-restricted access). A similar trend in SSR unit size abundance was observed with the random sample from the total database (Table 3). From a total of 343 microsatellites sampled, 98 (28.57\%) were mononucleotide, 131 (38.19\%) dinucleotide, 108 (31.49\%) trinucleotide, and 6 $(1.74 \%)$ either tetra or hexanucleotide. No pentanucleotide motifs were detected.

The distribution of the number of repeats across the SSR motif categories analyzed is presented in Figure 2 and Table 2. In general, for any of the SSR motif categories, the peak of the distribution was at the lowest number of repeats. Approximately $48 \%$ of monomeric microsatellites were of 10 to 11 repeats, whereas $59 \%$ of dimeric microsatellites were between 6 and 9 repeats, and $89.6 \%$ of trimeric

Table 2 - Occurrence of individual motif categories among the set of 20,530 microsatellites from the total Eucalyptus EST FORESTs database*.

\begin{tabular}{|c|c|c|c|c|c|c|c|c|c|c|c|c|c|c|}
\hline \multirow{3}{*}{$\begin{array}{l}\text { Number } \\
\text { of repeats }\end{array}$} & \multicolumn{14}{|c|}{ Category } \\
\hline & \multicolumn{2}{|c|}{ Monomeric } & \multicolumn{2}{|c|}{ 2-mer } & \multicolumn{2}{|c|}{ 3-mer } & \multicolumn{2}{|c|}{ 4-mer } & \multicolumn{2}{|c|}{5 -mer } & \multicolumn{2}{|c|}{ 6-mer } & \multicolumn{2}{|c|}{ Total } \\
\hline & $\#$ & $\%$ & $\#$ & $\%$ & \# & $\%$ & \# & $\%$ & $\#$ & $\%$ & \# & $\%$ & \# & $\%$ \\
\hline 5 & & & & & 2762 & 40.19 & 196 & 66.22 & 64 & 74.42 & 136 & 76.40 & 3158 & 15.38 \\
\hline 6 & & & 1555 & 20.46 & 1599 & 23.27 & 65 & 21.96 & 13 & 15.12 & 35 & 19.66 & 3267 & 15.91 \\
\hline 7 & & & 1178 & 15.50 & 1108 & 16.12 & 28 & 9.46 & 7 & 8.14 & 3 & 1.69 & 2324 & 11.32 \\
\hline 8 & & & 990 & 13.03 & 671 & 9.76 & 5 & 1.69 & & & 1 & 0.56 & 1667 & 8.12 \\
\hline 9 & & & 727 & 9.57 & 361 & 5.25 & 1 & 0.34 & & & 3 & 1.69 & 1092 & 5.32 \\
\hline 10 & 1585 & 28.82 & 635 & 8.36 & 208 & 3.03 & & & 2 & 2.33 & & & 2430 & 11.84 \\
\hline 11 & 1065 & 19.37 & 612 & 8.05 & 84 & 1.22 & 1 & 0.34 & & & & & 1762 & 8.58 \\
\hline 12 & 485 & 8.82 & 417 & 5.49 & 42 & 0.61 & & & & & & & 944 & 4.60 \\
\hline 13 & 365 & 6.64 & 359 & 4.72 & 15 & 0.22 & & & & & & & 739 & 3.60 \\
\hline 14 & 345 & 6.27 & 299 & 3.93 & 3 & 0.04 & & & & & & & 647 & 3.15 \\
\hline 15 & 299 & 5.44 & 222 & 2.92 & 2 & 0.03 & & & & & & & 523 & 2.55 \\
\hline 16 & 264 & 4.80 & 184 & 2.42 & 12 & 0.17 & & & & & & & 460 & 2.24 \\
\hline 17 & 161 & 2.93 & 104 & 1.37 & 1 & 0.01 & & & & & & & 266 & 1.30 \\
\hline 18 & 111 & 2.02 & 100 & 1.32 & & & & & & & & & 211 & 1.03 \\
\hline 19 & 107 & 1.95 & 51 & 0.67 & 1 & 0.01 & & & & & & & 159 & 0.77 \\
\hline 20 & 108 & 1.96 & 46 & 0.61 & & & & & & & & & 154 & 0.75 \\
\hline $21-30$ & 310 & 5.64 & 98 & 1.29 & 1 & 0.01 & & & & & & & 409 & 1.99 \\
\hline $31-40$ & 145 & 2.64 & 8 & 0.11 & & & & & & & & & 153 & 0.75 \\
\hline $41-50$ & 66 & 1.20 & 8 & 0.11 & 2 & 0.03 & & & & & & & 76 & 0.37 \\
\hline $51-60$ & 47 & 0.85 & 3 & 0.04 & & & & & & & & & 50 & 0.24 \\
\hline$>60$ & 36 & 0.65 & 3 & 0.04 & & & & & & & & & 39 & 0.19 \\
\hline $\begin{array}{l}\text { Total/ } \\
\text { category }\end{array}$ & 5499 & & 7599 & & 6872 & & 296 & & 86 & & 178 & & 20,530 & \\
\hline$\%$ & 26.79 & & 37.01 & & 33.47 & & 1.44 & & 0.42 & & 0.87 & & 100.00 & \\
\hline An & 14.81 & & 9.76 & & 6.39 & & 5.49 & & 5.43 & & 5.31 & & $7.87 \pm$ & 3.80 \\
\hline As & 14.81 & & 19.53 & & 19.16 & & 21.97 & & 27.15 & & 31.89 & & $22.42 \pm$ & 6.15 \\
\hline
\end{tabular}

*Complementary information on the individual motifs is available in the Appendix Table (upon request or at the site https://forests.esalq.usp.br.). An: Average number of repeats $=$ R. As: Average size $(b p)=$ R X motif category. 


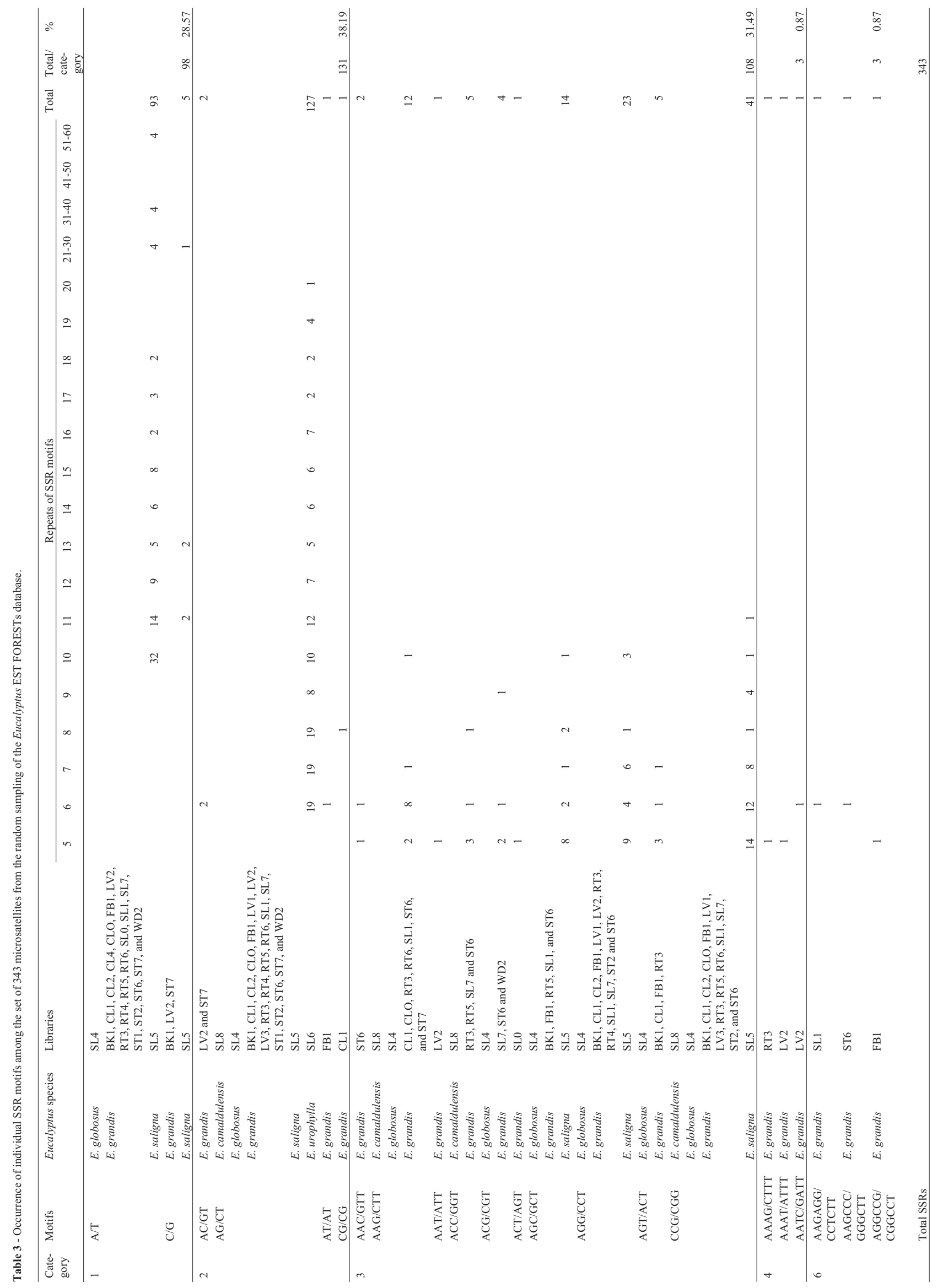




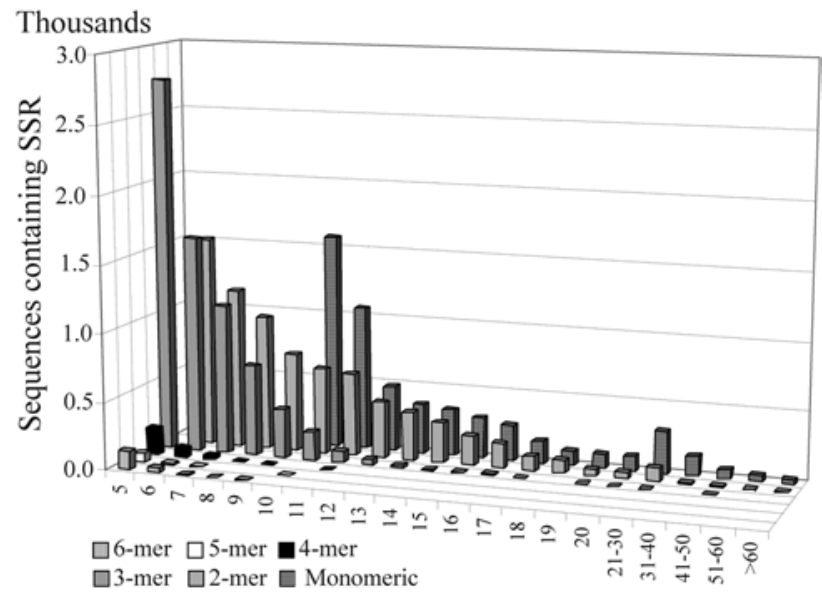

Figure 2 - Distribution of the number of repeats across the SSR motif categories detected in the 20,530 microsatellites found in the Eucalyptus EST FORESTs database.

microsatellites were of 5, 6 or 7 repeats. Eighty-eight to $96 \%$ of the tetra, penta or hexameric SSRs were of 5 to 6 repeats. The greater the complexity of the microsatellite motifs analyzed, the smaller the number of repeats found. The average number of repeats varied from 14.81 for monomeric, 9.76 for dimeric, 6.39 for trimeric, to $5.41 \pm$ 0.09 for tetra, penta or hexameric SSRs. The average number of repeats across all motifs analyzed was $7.87 \pm 3.80$. This has led to rather small microsatellites sizes within the Eucalyptus EST sequences. The average size of a microsatellite across all categories of SSR motifs was $22.42 \pm 6.15 \mathrm{bp}$. However, the microsatellite sizes varied from 14.8 (monomeric), 19.4 (dimeric and trimeric), 22.0 (tetrameric), 27.2 (pentameric) to $31.9 \mathrm{bp}$ (hexameric SSRs).

The occurrence of individual SSR motifs among the set of 20,530 microsatellites, which were found in the 71,115 Eucalyptus EST sequences (hierarchically grouped into distinct libraries) is summarized in the Appendix Table. Regarding monomeric SSRs, the A/T motif was more common $(22.57 \%)$ than the $\mathrm{G} / \mathrm{C}$ one, which represented $4.22 \%$ abundance. Among dimeric SSRs, the AG/CT motif was by far the most common one (35.13\%); AC/GT, $\mathrm{AT} / \mathrm{AT}$ and $\mathrm{CG} / \mathrm{CG}$ all together were represented at low abundance (from 0.18 to $1.03 \%$ of the total). The mostfrequent trimeric microsatellite motifs were CCG/CGG (12.81\%), AGG/CCT (7.01\%), AAG/CTT (4.78\%), $\mathrm{AGC} / \mathrm{GCT}(3.49 \%)$ and ACG/CGT $(2.13 \%)$. In general, considering the low-abundant tetra, penta and hexanucleotide motifs (with abundance varying from 0.044 to $0.22 \%$ ), the most frequent SSR motifs were AGGT/ACCT, AGCG/CGCT, AGGG/CCCT and AAGG/CCTT (tetrameric), ACACG/CGTGT, AGAGG/CCTCT, AAACC/ GGTTT, AAGCC/GGCTT and AATCG/CGATT (pentameric), AGGCCG/CGGCCT, AGAGGG/CCCTCT, AGCCCC/GGGGCT, AGCGGT/ACCGCT, AACGGC/ GCCGTT, AGCGGC/GCCGCT, AGGCGG/CCGCCT and AAGAGG/CCTCTT (hexameric).

Table 3 summarizes the occurrence of individual SSR motifs among the set of 343 microsatellites, which were found in the random sample of 1,217 Eucalyptus ESTs. The results were rather similar to the ones observed when analyzing the total database. Of the two monomeric SSRs detected, the $\mathrm{A} / \mathrm{T}$ motif was much more common (27.11\%) than the $\mathrm{G} / \mathrm{C}$ one, which represented only $1.46 \%$ abundance. Among dimeric SSRs, the AG/CT motif was by far the most common (37.03\%), whereas AC/GT, AT/AT and $\mathrm{CG} / \mathrm{CG}$ all together were represented at low abundance (from 0.29 to $0.58 \%$ of the total). The most frequent trimeric microsatellite motifs were CCG/CGG (11.95\%), AGG/CCT (6.71\%), AGC/GCT (4.08\%), AAG/CTT (3.50\%), and ACG/CGT, ACC/GGT or AGT/ACT (varying from 1.17 to $1.46 \%$ ). Differences between the two datasets were observed considering the low-abundant tetra nucleotide (with abundance of $0.87 \%$ ). The tetrameric SSR motifs detected (AAAG/CTTT, AAAT/ATTT, and AATC/ GATT) were not amongst the most common ones observed in the complete database. However, the hexameric SSRs detected (AGGCCG/CGGCCT, and AAGAGG/CCTCTT, AAGCCC/GGGCTT), were present with similar abundance in both datasets.

The distribution of the relative position (5' to 3' end) of microsatellites along a particular SSR containing sequence was analyzed in the random sample of the Eucalyptus EST database (Table 4). From a total of 343 SSRs, approximately $160(46.65 \%)$ of the microsatellites were found closest to the start of the EST sequence (between 0

Table 4 - Distribution of the relative position (5' to 3' end) of microsatellites along Eucalyptus EST sequences containing SSRs in the random sampling of the database.

\begin{tabular}{|c|c|c|c|c|c|c|}
\hline & \multicolumn{5}{|c|}{ Relative position (in terms of proportion of the total EST sequence length)* } & \multirow{3}{*}{$\begin{array}{l}\text { Total number of } \\
\text { SSR analyzed }\end{array}$} \\
\hline & $0.00-0.15$ & $0.151-0.35$ & $0.351-0.65$ & $0.651-0.85$ & $0.851-1.00$ & \\
\hline & I & IM & M & $\mathrm{ME}$ & $\mathrm{E}$ & \\
\hline Number of SSRs & 160 & 80 & 57 & 18 & 28 & 343 \\
\hline$\%$ & 46.65 & 23.32 & 16.62 & 5.25 & 8.16 & 100.00 \\
\hline
\end{tabular}

$* \mathrm{I}=$ close to the beginning of the sequence ( 5 '-end position); $\mathrm{IM}=$ between the start and the middle; $\mathrm{M}=$ middle; $\mathrm{ME}=$ between the middle and the end; $\mathrm{E}=$ close to the end of the sequence (3'-end position). 
and $15 \%$ of total sequence length, starting at the 5 ' end), whereas $28(8.16 \%)$ were located closest to the $3^{\prime}$ ' end (between 85.1 to $100 \%$ of the total sequence length). The remaining 155 SSRs $(45.19 \%)$ were distributed intermediately along the EST sequences.

The random sample of 265 SSR-containing EST sequences was characterized according to their gene function, considering previous information on automatic BLASTX homology comparisons, available at the FORESTs database. The information on gene ontology is available in Table 5. Fifty-two of the total SSR-containing EST sequences $(19.62 \%)$ produced no hits with any known proteins sequences, whereas 87 (32.83\%) had homology with hypothetical, putative, unknown or Arabidopsis thaliana expressed proteins (but with unknown function). A total of $126(47.55 \%)$ EST sequences containing microsatellites were homologous to proteins with known biological function. From these, 47 (17.74\%) were classified as involved in biological processes such as cell communication, embryonic development, cellular physiological process, metabolism, pathogenesis, response to external stimulus, or of unknown physiological process. Of the biological function related proteins, which had homology with Eucalyptus EST sequences bearing SSRs, the ones associated with metabolism were the most frequent within this category (a total of 32 , or $12.08 \%$ ). The metabolism proteins were associated with biosynthesis processes, with metabolism of carbohydrate, nucleobase, nucleoside, nucleotide and nucleic acid, organic acid, oxygen and reactive oxygen species, phosphorus, and protein, with catabolism, and electron transport.

The majority of the SSR-containing EST sequences (a total of 69 , or $26.04 \%$ ) were homologous with proteins of molecular functions, such as binding (30), catalytic (29), transporter (6), chaperone (1), enzyme regulator (1), signal transducer (1), and structural molecule (ribosome) activity (1). Of the binding activity category homologous proteins, the ones associated with nucleic acid binding (17) and metal ion binding (7) were the most frequent. In the catalytic proteins group, the following categories were detected: transferase (9), hydrolase (7), kinase (4), lyase (2), small protein activating enzyme (2), helicase (1), isomerase (1), and oxidoreductase (1) activity.

The last category of proteins homologous to EST sequences containing SSRs were the cellular component ones (a total of 10, representing 3.77\%), which were intracellular (associated with the mitochondrion, or as ribonucleoprotein complex), integral to membrane (proton-transporting two-sector ATPase complex), with extracellular or unlocalized functions.

\section{Discussion}

Microsatellites or SSRs are considered preferred genetic markers in plants for several reasons, such as their relative abundance and random distribution on the genome,

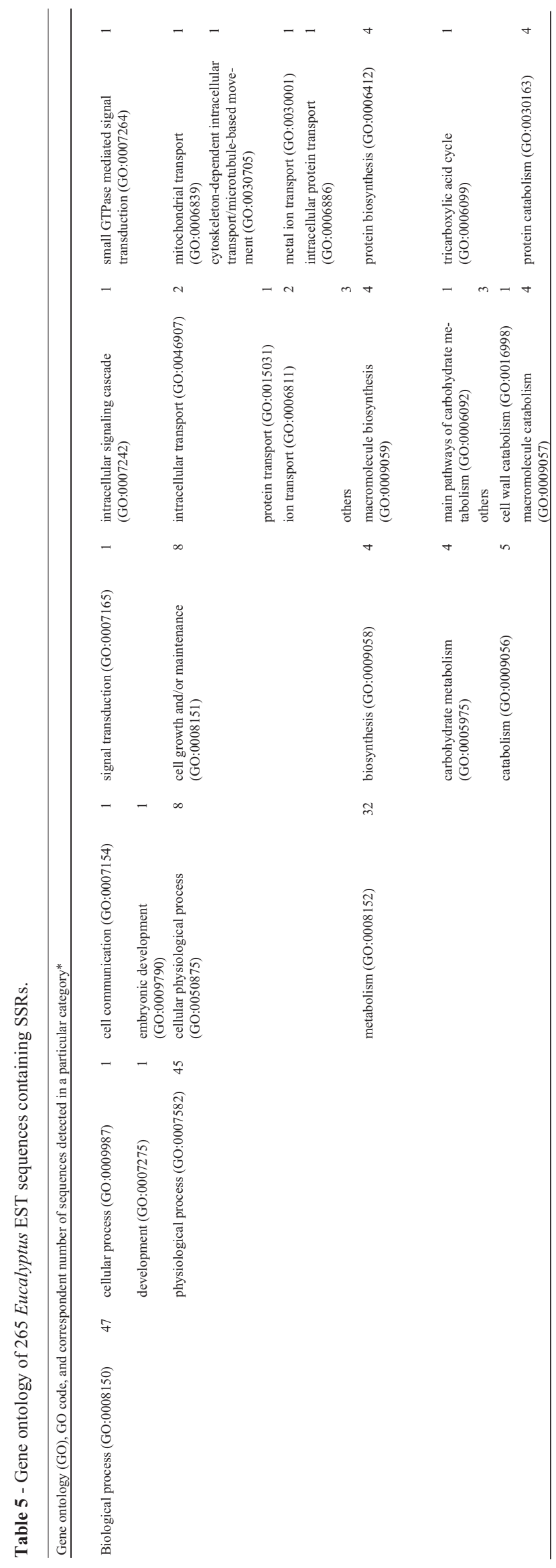




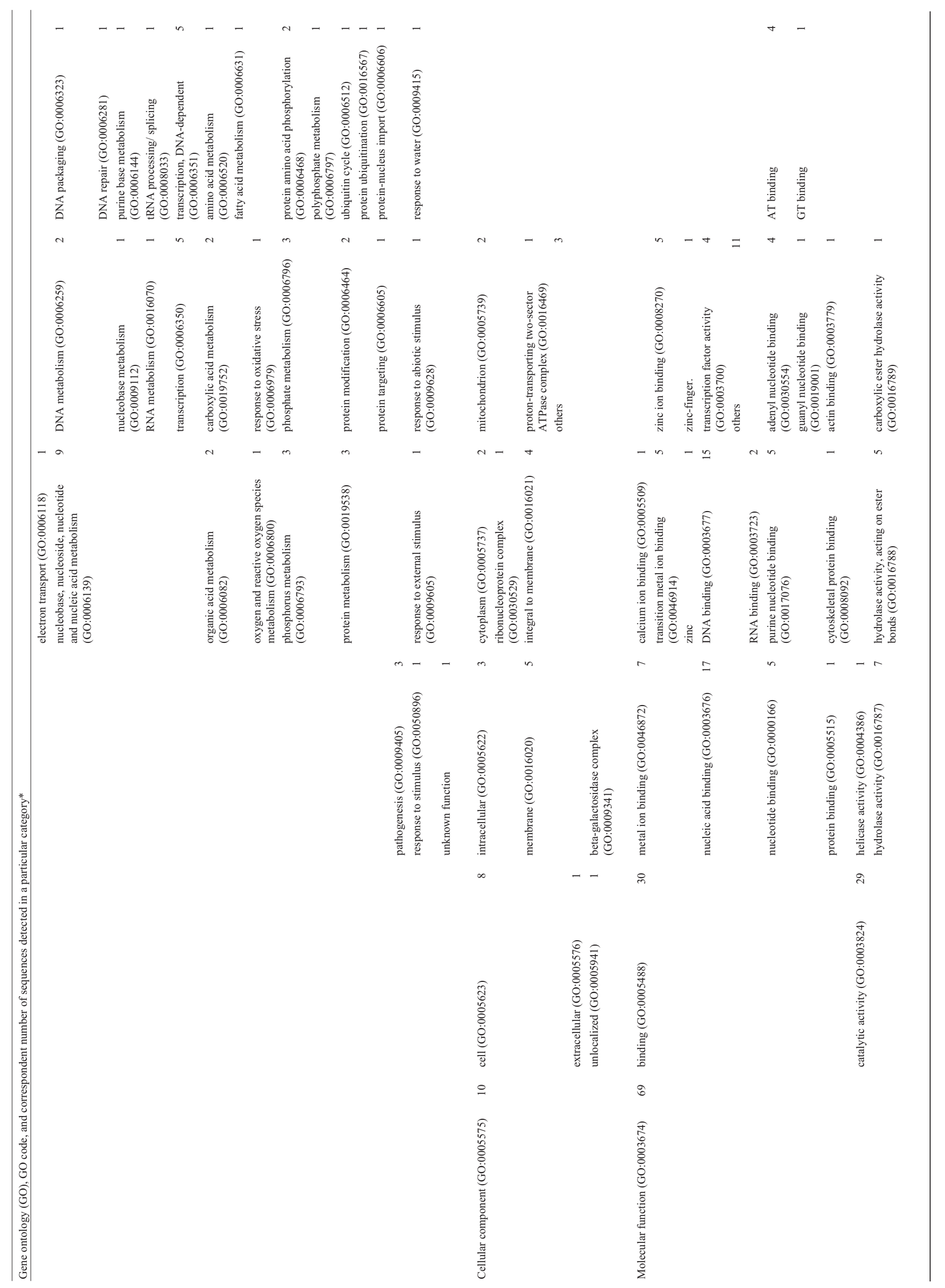




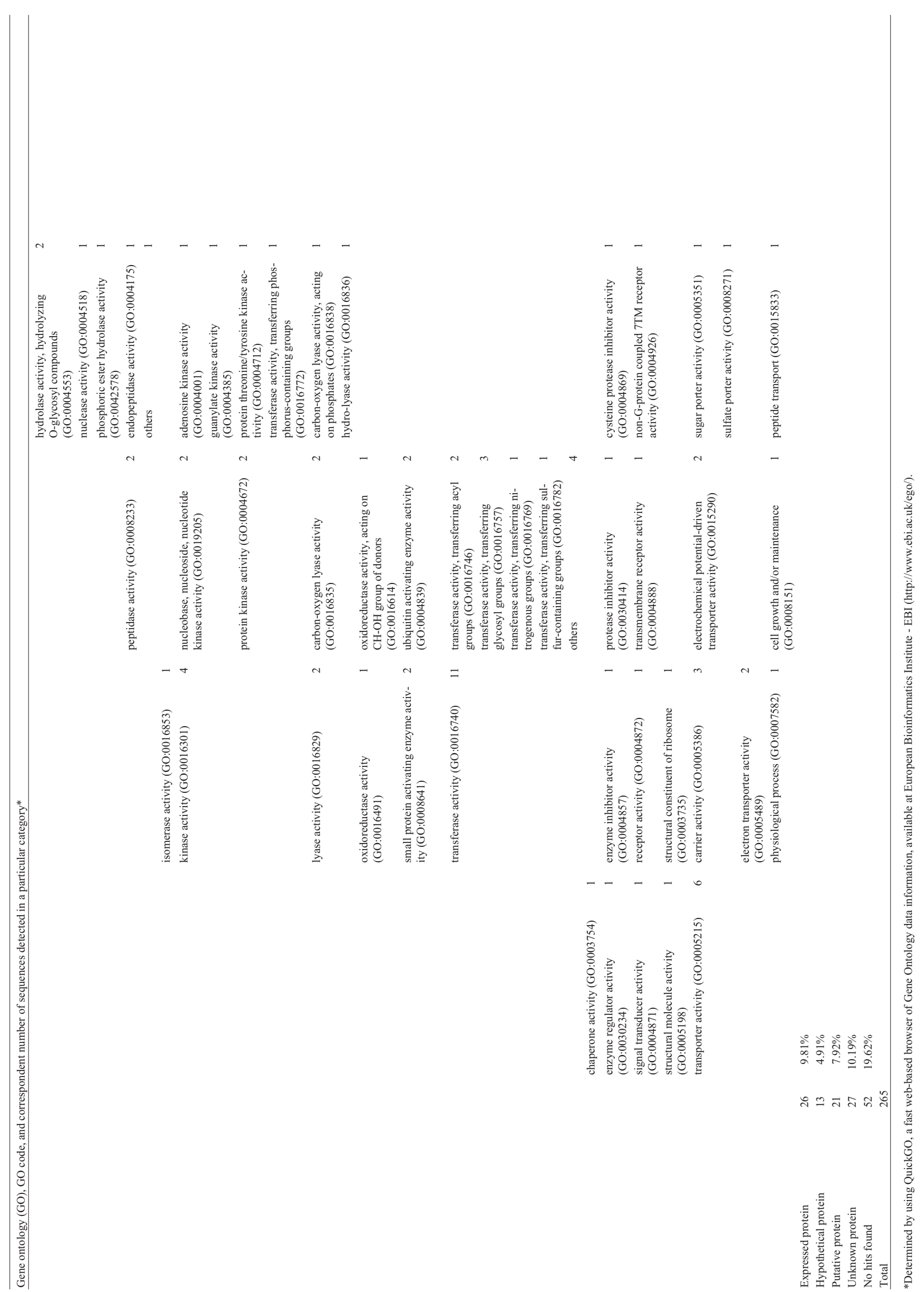


high polymorphism (multiallelic nature), codominant inheritance in a Mendelian fashion, somatic stability, selective neutrality and simple detection by PCR using two unique primers flanking the microsatellites (Morgante and Olivieri, 1993; Powell et al., 1996). However, the use of SSR-markers is not as extensive or widespread as desired, considering that the standard methods for developing SSR-markers are time consuming and labor-intensive, requiring the creation of small-insert libraries, hybridization with tandemly repeated oligonucleotides and sequencing of candidate clones (Brondani et al., 1998).

The development of EST-derived microsatellite markers has advantages over the traditional method as they can be considered freely obtained by-products from EST sequences databases, with minimal development costs. Thus, for reducing time and development costs, the main goal of our research was to search for SSRs in the Eucalyptus EST FORESTs database (using software for mining SSR-motifs). The other objective was to characterize and catalog the Eucalyptus EST-derived SSR-motifs according to their abundance, information content, and genomic distribution. With this objective, we created a database for cataloging Eucalyptus EST-derived SSR, and developed a bioinformatics tool, named Satellyptus, for finding and analyzing microsatellites in the Eucalyptus EST database.

The frequency of occurrence of SSRs on expressed sequences is considered, in general, low due to an associated lower polymorphism of coding regions in contrast to non-coding ones. The occurrence of particular microsatellites motifs and repeats (especially the non-trimeric ones) could have implications on how the gene coding region is transcribed, due to risks of frameshift mutations that may occur when other microsatellites classes alternate in size of one unit (Metzgar et al., 2000).

However, out of a total of 71,115 Eucalyptus EST sequences analyzed, 20,530 microsatellites were detected. This resulted in the identification of one microsatellite for every four examined sequences or a total abundance of $29 \%$, which is considered relatively high according to the literature available. For example, Thiel et al. (2003) have detected a ratio of one microsatellite identified out of every 12 barley (Hordeum vulgare) EST sequences (abundance of $8.2 \%$ ). The SSR abundance detected on the Eucalyptus ESTs database is still in disagreement with that observed for other plant species, such as grapes and sugarcane, for which the frequency and abundance ratio varied from $3.1 \%$ (1:32) to $2.9 \%$ (1:34), respectively (Scott et al., 2000; Cordeiro et al., 2001)

Comparing the average distance $(\mathrm{kb})$ between two SSRs in the Eucalyptus ESTs with information of microsatellites on cDNA libraries from other plant species, the frequency of SSR detected on Eucalyptus ESTs was found to be very high. For example, while an Eucalyptus EST-contained microsatellite was detected every $2.7 \mathrm{~kb}$, the following higher distance values between two SSRs were observed: 3.4 for rice, 6.3 for barley, 8.1 for corn, 7.4 for soybean, 11.1 for tomato, 13.8 for Arabidopsis, and 20 for cotton (Cardle et al., 2000; Thiel et al., 2003).

Despite the high frequency of microsatellites on $\mathrm{Eu}$ calyptus ESTs, only approximately 45\% (or 9,277 SSR-containing sequences) will be appropriate for the design of flanking primers aiming the development of microsatellite markers, since the remaining $54.8 \%$ of the EST sequences contained SSRs situated too close to either 5 ' or 3 'end of the sequences. Therefore, from a initial abundance ratio of 1:4, only one SSR every eight sequences could be effectively utilized for developing markers based on the amplification of the flanking region containing the SSR locus.

Considering the average size of any SSR motif detected on Eucalyptus ESTs, 97\% of the microsatellites were smaller than $20 \mathrm{bp}$, in concordance with that observed for other plant species (Thiel et al., 2003).

Of the categories of SSR motifs detected on Eucalyptus ESTs, the dimeric (37\%) and trimeric ones (33\%) predominate. The AG/CT motif in particular was the most frequent one $(35.15 \%)$ in the entire database, in agreement with the available literature on Eucalyptus microsatellites (Brondani et al., 2002). All the remaining dimeric motifs (AC/GT, AT/AT and CG/CG) were detected in low frequency/abundance. The number of motif repeats, however, interferes with the level of the marker's polymorphism. The highest level of polymorphism on Eucalyptus (22 alleles) was observed at a locus composed by a dinucleotide motif based on 15 AG repeats, whereas the lowest level (7 alleles) was detected with 18 AG repeats (Brondani et al., 2002). Similar results have been reported for Pinus (Echt et al., 1999), where longer repeats are associated with lower polymorphism. Despite the low frequency of AC motif repeats in plant genome (Morgante and Olivieri, 1993), the levels of polymorphism were similar for both AC and AG motifs in Eucalyptus (Brondani et al., 2002).

Repetitive motif structure did not seem to be an indicator of polymorphism for Eucalyptus. For eight imperfect and nine compound sequences characterized by Brondani et al. (2002), the genetic information content was high and similar to that observed for perfect repeats. Compound microsatellites were also detected within the same sequence of a particular Eucalyptus EST (13.6\% of the total SSR found), which is also reported for other plant species (Yaish and Pérez de la Vega, 2003).

Within the trimeric category, the CCG/CGG motif (12.81\%) was the most frequent detected on Eucalyptus ESTs. Other trimerics detected with relative importance were the AGG/CCT (7.00\%), AAG/CTT (4.78\%), AGC/GCT (3.49\%), and ACG/CGT (2.13\%), with levels of abundance similar to those reported in the literature (Thiel et al., 2003; Temnykh et al., 2000; Chin, 1996). The AAT/ATT motif appeared less often in Eucalyptus ESTs $(0.11 \%)$, probably because TAA variants code for stop 
codons, which could directly affect the protein synthesis (Chin, 1996).

In a unique initiative, we characterized a set of Eucalyptus SSR-containing ESTs by function, searching for any pattern of association of SSRs with specific gene classes. However, no specific pattern of association was detected. Besides, 54\% of the ESTs sequences containing SSRs had no homology with proteins of known function. In general, the SSRs detected in Eucalyptus ESTs were homologous to proteins involved with very distinct molecular functions (such as binding, catalytic, transporter, chaperone, enzyme regulator, signal transducer, and structural molecule (ribosome) activities), biological processes (especially the ones related with metabolism) and cell; the high frequency of SSR-containing sequences in the Eucalyptus EST database could somehow be interpreted as a paradox (Yaish and Pérez de la Vega, 2003). However, we postulated that these particular SSRs occupy certain positions in the coding regions that do not invalidate (or negatively affect) the activity of these gene products, which were found most frequently associated with vital metabolic pathways, processes or cellular components.

The outcomes of this research, as presented, could be considered strictly theoretical and rather speculative in nature, thus requiring further validation studies to achieve its most important objective: the development of SSR-based markers for Eucalyptus. Therefore, priority should be given to the next most important task of obtaining microsatellitebased markers suitable for marker-assisted Eucalyptus breeding programs.

The greatest obstacles for identification and comprehension of quantitative traits variation in Eucalyptus are the low throughput and the limited polymorphic information content of many molecular marker classes (such as RLFP) used for marker-assisted selection (Grattapaglia, 1999). Contrarily to such markers, the importance of microsatellite loci for Eucalyptus resides in the fact that they are extremely valuable for marker assisted breeding programs, as powerful tools in analyses of qualitative and quantitative trait loci. This is due to its highly polymorphic multiallelic nature. For example, from 50 microsatellite markers developed for E. grandis and E. urophyla, 717 alleles were identified in 32 individuals. The range of alleles per locus varied from 5 to 16 for E. urophylla and 4 to 17 to E. grandis (Brondani et al., 2002). They are also extremely important for fingerprinting, quality control of controlled crossing, monitoring of inbreeding and assessment of gene flow and genetic differentiation among populations (Powell et al., 1996; Chen et al., 1997)

The mapping of important candidate genes for lignification, based on microsatellite markers with alleles segregating from both parents, would constitute another important approach to understanding the molecular basis of the quantitative variation (Gion et al., 2000; Kirst et al., 2001).
Furthermore, microsatellites are highly transferable from genome to genome within species and, frequently, among species (Marques et al., 2002; Brondani et al., 2002). For instance, all seventy of the SSR marker loci developed for Eucalyptus were found fully transferable between E. grandis and E. urophylla and highly genetically informative. On average, $45 \%$ of the alleles observed were shared between the two species (Brondani et al., 2002). These findings establish the basis for fundamental research such as evolutionary studies and the use of synteny in the genetic analysis of the genus Eucalyptus as well. Considering that most of the Eucalyptus EST-consensus sequences were generated across libraries of distinct species (E. grandis, E. globosus, E. saligna and E. urophylla), enormous resources to test and implement related studies on genome synteny and evolution of Eucalyptus are available.

To attain the goal of developing such valuable microsatellite markers, our first initiative will be to improve the database by incorporating information on the design of flanking primers for codominant SSR loci. Validation studies are urged to develop and characterize a collection of EST-derived SSR-markers in terms of information content, genomic distribution, and transferability to related Eucalyptus species and to assess their potential for diversity analysis in a reference set of $E$. grandis cultivars.

\section{Acknowledgments}

We would like to thank Centro Virtual Omega / PROPP-UNESP for facilitating the annotation tasks through financing the computer hardware needed. We would also like to thank the FORESTs Consortium, which provided us with access to its database.

\section{References}

Brondani RPV, Brondani C and Grattapaglia D (2002) Towards a genus-wide reference linkage map for Eucalyptus based exclusively on highly informative microsatellite markers. Mol Genet Genomics 267:338-347.

Brondani RPV, Brondani C, Tarchini R and Grattapaglia D (1998) Development, characterization and mapping of microsatellite markers in Eucalyptus grandis and E. urophylla. Theor Appl Genet 97:816-827.

Cardle L, Ramsay L, Milbourne D, Macaulay M, Marshall D and Waugh R (2000) Computational and experimental characterization of physically clustered simple sequence repeats in plants. Genetics 156:847-857.

Chen X, Temnykh S, Xu Y, Cho YG and McCouch SR (1997) Development of a microsatellite framework map providing genome wide coverage in rice (Oryza sativa L.). Theor Appl Genet 95:553-567.

Chin ECL (1996) Maize simple repetitive DNA sequences: Abundance and allele variation. Genome 39:866-873.

Cordeiro GM, Casu R, McIntyre CL, Manners JM and Henry RJ (2001) Microsatellite markers from sugarcane (Saccharum 
spp.) ESTs cross transferable to erianthus and sorghum. Plant Sci 160:1115-1123.

Echt CS, Vendramim GG, Nelson CD and Marquardt P (1999) Microsatellite DNA as shared genetic markers among conifer species. Can J For Res 29:365-371.

Eujayl I, Sorrells ME, Baum M, Wolters P and Powell W (2002) Isolation of EST-derived microsatellite markers for genotyping the A and B genomes of wheat. Theor Appl Genet 104:399-407.

Gion JM, Rech P, Grima-Pettenati J, Verhaegen D and Plomion C (2000) Mapping candidate genes in Eucalyptus with emphasis on lignification genes. Mol Breed 6:441-449.

Grattapaglia D (1999) Molecular breeding of Eucalyptus - State of the art, operational applications and technical challenges. In: Jain SM (ed) Molecular Markers and Genome Mapping in Woody Plants. v 1. Kluwer Academic Publishers, Dordrecht, pp 451-474.

Hackauf B and Wehling P (2002) Identification of microsatellite polymorphisms in an expressed portion of the rye genome. Plant Breed 121:17-25.

Huang X and Madan A (1999) CAP3: A DNA sequence assembling program. Genome Research 9:868-877.

Kirst M, Kirst EM, Gurgel G, Myburg AA and Whetten RW (2001) Gene discovery in Eucalyptus grandis xylem. Plant and Animal Genome IX (San Diego) Abstract P502.

Marques CM, Brondani RPV, Grattapaglia D and Sederoff R (2002) Conservation and synteny of SSR loci and QTLs for vegetative propagation in four Eucalyptus species. Theor Appl Genet 105:474-478.
Metzgar D, Bytof J and Wills C (2000) Selection against frameshift mutations limits microsatellite expansion in coding DNA. Genome Res 10:72-80.

Morgante M and Olivieri AM (1993) PCR-amplified microsatellites as markers in plant genetics. Plant J 3:175-182.

Powell W, Machray GC and Provan J (1996) Polymorphism revealed by simple sequence repeats. Trends Plant Sci 1:215222.

Ramsay L, Macaulay M, Ivanissevich DS, MacLean K, Cardle L, Fuller J, Edwards KJ, Tuvesson S, Morgante M, Massari A, Maestri E, Marmiroli N, Sjakste T, Ganal M, Powell W and Waugh R (2000) A simple sequence repeat-based linkage map of barley. Genetics 156:1997-2005.

Scott KD, Eggler P, Seaton G, Rossetto M, Ablett EM, Lee LS and Henry RJ (2000) Analysis of SSRs derived from grape ESTs. Theor Appl Genet 100:723-726.

Struss P and Plieske J (1998) The use of microsatellite markers for detection of genetic diversity in barley populations. Theor Appl Genet 97:308-315.

Temnykh S, Park WD, Ayres N, Cartinhour S, Hauck N, Lipovich L, Cho YG and McCouch SR (2000) Mapping and genome organization of microsatellite sequences in rice (Oriza sativa L.) Theor Appl Genet 100:692-712.

Thiel T, Michalek W, Varshney RK and Graner A (2003) Exploiting EST databases for the development and characterization of gene-derived SSR-markers in barley (Hordeum vulgare L.). Theor Appl Genet 106:411-422.

Yaish MWF and Pérez de la Vega M (2003) Isolation of (GA) microsatellite sequences and description of a predicted MADS-box sequence isolated from common bean (Phaseolus vulgaris). Genet Molec Biol 26:337-342.

Associate Editor: Marie Anne Van Sluys 